\title{
Gravitational Wave Radiated by a Collapsing Ellipsoid
}

L. G. de Almeida*ab

${ }^{a}$ Universidade Federal do Acre

Campus Cruzeiro do Sul, Acre, Brazil

${ }^{b}$ Centro Brasileiro de Pesquisas Físicas

Rua Dr. Xavier Sigaud 150, 22290-180, Rio de Janeiro, RJ, Brazil

E-mail: <lgalmeida@gmail.com

\section{S. B. Duarte}

Centro Brasileiro de Pesquisas Físicas

Rua Dr. Xavier Sigaud 150, 22290-180, Rio de Janeiro, RJ, Brazil

E-mail: sbdecbpf.br

\section{Portes Jr}

Centro Federal de Educação Tecnológica do Rio de Janeiro

Av Maracanã 249, 20271-110, Rio de Janeiro, RJ, Brazil

E-mail: portes@mail.com

\section{H. Rodrigues ${ }^{a b}$}

${ }^{a}$ Department of Physics, San Diego State University 5500 Campanile Drive, San Diego, CA 92182, USA

${ }^{b}$ Centro Federal de Educação Tecnológica do Rio de Janeiro Av Maracanã 249, 20271-110, Rio de Janeiro, RJ, Brazil

E-mail: hargesciences.sdsu.edu

In this work we present a simplified effective description of the final state of the gravitational collapse of a uniformly rotating protoneutron star, approximated by a compressible homogeneous triaxial ellipsoid. The generation of gravitational waves is treated within the weak field limit. The method can be applied to estimate the intensity of the radiated gravitational waves during the core collapse.

XXXIV edition of the Brazilian Workshop on Nuclear Physics,

5-10 June 2011

Foz de Iguaçú, Paraná, Brazil

\footnotetext{
* Speaker.
} 


\section{Introduction}

At the end of the evolution of massive stars a dense core composed basically of the iron group nuclei is formed. At this stage the star core becomes dynamically unstable against gravitation, due to processes such as photo dissociation of nuclei and electron capture, which significantly reduce the hydrostatic pressure. So, inevitably the core collapses. During the gravitational collapse up to $99 \%$ of the available gravitational binding energy is expended on the production of neutrinos ( $\sim 10^{53} \mathrm{erg}$ ) which initially escape from the collapsing core, and only around $1 \%$ is converted into kinetic energy of the ejected star material, gravitational waves and electromagnetic radiation. The gravitational collapse can lead to the formation of a neutron star, a black hole, or even the complete disruption of the star, depending on the value of the star core mass prior to the gravitational collapse.

Neutron stars are fast rotating systems whose periods of revolution can be as low as few milliseconds. The rotation causes nonsphericity and part of the gravitational binding energy can be expended in the form of gravitational waves.

The complete analysis of the gravitational collapse of a supernova core and the formation of a neutron star is a very complicated task only achieved through complex numerical codes. On the other hand, these calculations require the knowledge of a large number of physical inputs which have in general large uncertainties. In this context, it may be interesting to formulate simplified models based on reasonable assumptions which however are able to capture the basic aspects of the corresponding problem.

In this work we present such a type of simple description of the final state of the gravitational collapse of a uniformly rotating star core. The stellar matter is treated as a cold compressible homogeneous fluid with a barotropic zero-temperature equation of state $\varepsilon=\varepsilon(p)$, where $\varepsilon$ is the energy density and $p(\rho)$ is the pressure. We solve numerically the equations of motion for the semi-axes of the core star, taken as the dynamic variables of the collapsing system with rotation.

An effective Lagrangian of the ellipsoid is constructed incorporating the kinetic energy associated with the radial motion, the rotational energy, the gravitational energy and the internal energy, written in terms of the semi-axes of the ellipsoid and their time derivatives. We then solve numerically the equations of motion for the three semi-axes of the protoneutron star, for a given initial condition. The generation of gravitational waves is treated within the weak field limit approximation, providing two additional equations, one describing the rate of the radiated energy and one describing the rate of the angular momentum loss. The method can be used to estimate the intensity of the radiated gravitational waves.

\section{The Model}

The dynamics of a rigidly rotating neutron star is approximated by a compressible homogeneous triaxial ellipsoid. We construct an effective Lagrangian $L=K-W-U_{\text {rot }}-U_{\text {int }}$, with $K$ being the translational kinetic energy, $W$ the gravitational potential energy, $U_{i n t}$ the internal energy, and $U_{r o t}$ the rotational kinetic energy. All quantities are written in terms of the semi-axes of the ellipsoid $a_{1}, a_{2}$, and $a_{3}$, and their respective time derivatives. 
The gravitational potential energy of the triaxial ellipsoid is given by

$$
W=-\frac{3}{10} G M^{2} \frac{A}{a_{1} a_{2} a_{3}},
$$

where $A$ is defined by

$$
A=\sum_{i=1}^{3} A_{i} a_{i}^{2}
$$

where

$$
A_{i}=a_{1} a_{2} a_{3} \int_{0}^{\infty} \frac{d \zeta}{\Delta\left(a_{i}^{2}+\zeta\right)}
$$

with

$$
\Delta^{2}=\left(a_{1}^{2}+\zeta\right)\left(a_{2}^{2}+\zeta\right)\left(a_{3}^{2}+\zeta\right)^{1 / 2} .
$$

The coefficients $A_{i}$ satisfy the relationship $\sum_{i=1}^{3} A_{i}=2$.

The flow of the fluid is governed by the continuity equation. For an irrotational flux the translational kinetic energy associated with the internal motion of the fluid, measured the rotating frame, is given by the simple form

$$
K=\frac{1}{10} M\left(\dot{a}_{1}^{2}+\dot{a}_{2}^{2}+\dot{a}_{3}^{2}\right) .
$$

The rotational energy measured in the rest frame fixed at the ellipsoid center is given by

$$
U_{r o t}=\frac{5}{2} \frac{J^{2}}{M\left(a_{1}^{2}+a_{2}^{2}\right)} .
$$

The equations of motion for the three generalized coordinates extracted from the Lagrangian of the system read

$$
\begin{aligned}
& \ddot{a}_{1}=-\frac{3}{2} \frac{G M}{a_{2} a_{3}} A_{1}+\frac{25 J^{2}}{M^{2}} \frac{a_{1}}{\left(a_{1}^{2}+a_{2}^{2}\right)^{2}}+\frac{20 \pi}{3 M} P a_{2} a_{3}, \\
& \ddot{a}_{2}=-\frac{3}{2} \frac{G M}{a_{1} a_{3}} A_{2}+\frac{25 J^{2}}{M^{2}} \frac{a_{2}}{\left(a_{1}^{2}+a_{2}^{2}\right)^{2}}+\frac{20 \pi}{3 M} P a_{1} a_{3},
\end{aligned}
$$

and

$$
\ddot{a}_{3}=-\frac{3}{2} \frac{G M}{a_{1} a_{2}} A_{3}+\frac{20 \pi}{3 M} P a_{1} a_{2},
$$

where $P$ is the fluid pressure.

Let us assume that the source of gravitational waves has a mass quadrupole moment tensor $D_{\alpha \beta}$. The rate of energy loss due to the emission of gravitational waves in the weak field limit is given by [2]

$$
\frac{d E}{d t}=-\frac{G}{45 c^{5}}\left(\frac{\partial^{3} D_{\alpha \beta}}{\partial t^{3}}\right)^{2} .
$$

Thus, in the approximation of quadrupole radiation the power emitted by an ellipsoid rotating rigidly about the $x_{3}$-axis at a single frequency $\Omega$ is given by $[4,5,6]$

$$
-\frac{d E}{d t}=\frac{32}{125} \frac{G M^{2} \Omega^{6}}{c^{5}}\left(a_{1}^{2}-a_{2}^{2}\right)^{2} .
$$


On the other hand, the rate of angular momentum loss can be also determined as [3]

$$
\frac{d J_{i}}{d t}=-\frac{2 G}{45 c^{5}} \varepsilon_{i j k} \frac{\partial^{2} D_{j l}}{\partial t^{2}} \frac{\partial^{3} D_{k l}}{\partial t^{3}},
$$

$J_{i}$ being the $i$-th component of the angular momentum $\vec{J}$ and $\varepsilon_{i j k}$ the Levi-Civita symbol. If the rotation is about the axis of symmetry then only the $x_{3}$-axis component is nonzero, and in this case the loss rate can be readily calculated

$$
\frac{d J}{d t}=-\frac{32}{125} \frac{G M^{2} \Omega^{5}}{c^{5}}\left(a_{1}^{2}-a_{2}^{2}\right)^{2} .
$$

\section{Results and Conclusions}

We solve numerically the equations of motion for the three semi axes of the protoneutron star for a given initial equilibrium condition. The generation of gravitational waves is treated within the weak field limit approximation, providing two additional equations: one describing the rate of the radiated energy and other one describing the rate of the angular momentum loss.

Here we assume the initial equilibrium configuration of the protoneutron star is a homogeneous triaxial ellipsoid, composed of purely hadronic matter. The mass, the eccentricity of the meridional sections and the semi-axes are determined by the gravitational meta-stable initial condition, prescribed for a given mass $M$ and total angular momentum $J$.

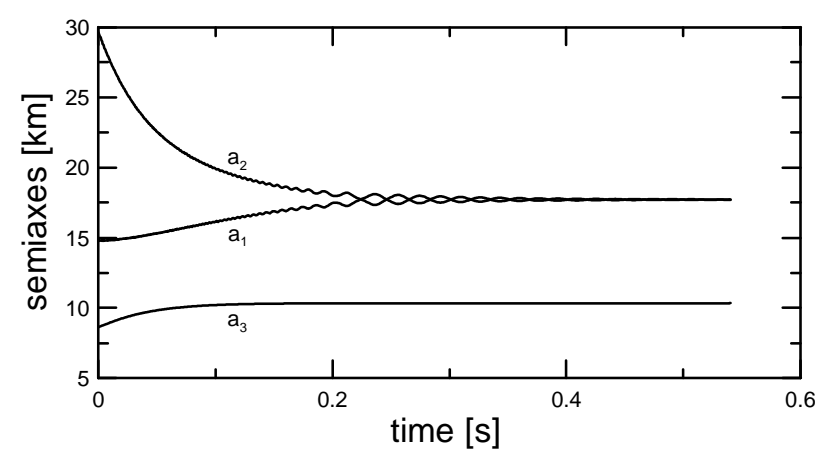

Figure 1: Time evolution of the three semi-axes of the ellipsoidal protoneutron star.

The damping of oscillations is modeled phenomenologically by the terms [7]

$$
-\frac{\dot{a}_{i}}{\tau_{r e l}} \quad(i=1,3)
$$

which are added to the right-hand side of equations (2.7) and (2.9), respectively. The relaxation time $\tau_{\text {rel }}$ is scaled by

$$
\tau_{\text {rel }}=\alpha \tau_{J},
$$


$\alpha$ being a constant factor and $\tau_{J}$ the Jeans characteristic time, defined by

$$
\tau_{J}=2 \pi \sqrt{\frac{a_{1}^{2} a_{3}}{3 G m}},
$$

where $\omega=\sqrt{4 \pi G \rho}$ is the characteristic frequency of the system.
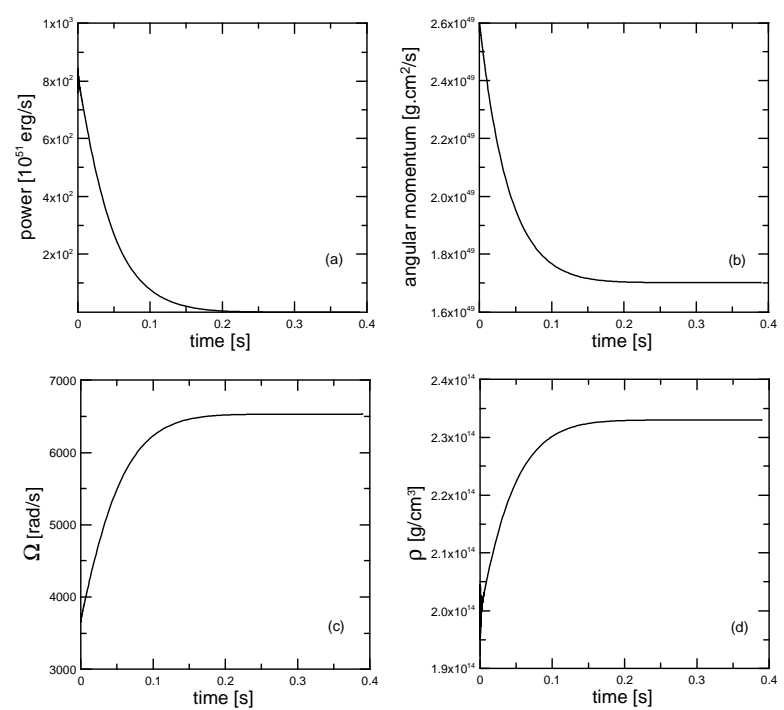

Figure 2: Power of the emitted gravitational waves (part-a); angular momentum time evolution (part-b); angular velocity as a function of time (part-c); and the time evolution of the neutron star density (part-d).

Figure 1 shows the time evolution of the three semi axes of a fast rotating $1.6 M_{\odot}$ protoneutron star. The initial angular momentum of the protoneutron star is $J=2.0 \times 10^{49} \mathrm{~g} . \mathrm{cm}^{2} . \mathrm{s}^{-1}$. As we can see, the emission of gravitational waves leads the protoneutron star to reach an oblate esferoidal form as the final equilibrium configuration, restoring the axial symmetry.

In part-a of the panel of the Figure 2 the power of the radiated gravitational wave is depicted as a function of time. We see that the power of the emitted gravitational waves decreases as the axial symmetry of the rotating neutron star is restored. In part-b one shows the time evolution of the angular momentum of the neutron star. In part-c we can see the time evolution of the angular velocity of the neutron star. In spite of the decrease in the angular momentum of the neutron star due to the emission of gravitational waves, we observe a significant increase in the angular velocity of the neutron star. This increasing is caused by the variation of the moment of inertia of the neutron star. Finally, part-d shows the increase of the density of the neutron star.

In conclusion, in this work we have presented a simplified description of the gravitational waves generated by a rotating collapsing core. Starting from a triaxial form, the neutron star assumes the final form of a fast rotating oblate spheroid as a consequence of gravitational wave radiation. The final state of the initial ellipsoidal protoneutron star is a uniformly rotating oblate spheroid 
with period, radius, density and chemical composition typical of neutron stars. The method is meant to estimate the intensity of the radiated gravitational waves by neutron stars.

in a compact collapsing core.

\section{References}

[1] S. Chandrasekhar, Ellipsoidal Figures of Equilibrium, Yale Univ. Press, New Haven (1969).

[2] L. D. Landau, and E. M. Lifshitz, The Classical Theory of Fields, Pergamon Press, London (1975).

[3] P. C. Peters, Phys. Rev. 136, 1229 (1964).

[4] C. W. Chin, Phys. Rev. 139B, 761 (1965).

[5] W. Y. Chau, Astrophys. J 147, 664 (1967).

[6] H. Beltrami, and W. Y. Chau, Astrophys. Space Sci. 111, 335 (1985).

[7] D. Lynden-Bell, MNRAS 136, 101 (1967). 